\title{
ПОЛУЧЕНИЕ МАТРИЧНОГО МАТЕРИАЛА НА ОСНОВЕ АЛЮМИНАТНОГО ПЕРОВСКИТА, ПРЕДНАЗНАЧЕННОГО ДЛЯ ИММОБИЛИЗАЦИИ АКТИНОИДОВ, МЕТОДОМ САМОРАСПРОСТРАНЯЮЩЕГОСЯ ВЫСОКОТЕМПЕРАТУРНОГО СИНТЕЗА
}

\author{
Долматов Олег Юрьевич 1 , \\ dolmatov@tpu.ru
}

\author{
Кузнецов Михаил Сергеевич 1 , \\ kms@tpu.ru
}

\author{
Семенов Андрей Олегович1, \\ semenov_ao@tpu.ru \\ 1 Национальный исследовательский Томский политехнический университет, \\ Россия, 634050, г. Томск, пр. Ленина, 30.
}

\begin{abstract}
Актуальность исследования обусловлена необходимостью разработки способа получения перспективных минералоподобных матричных материалов, предназначенных для иммобилизации наиболее опасных с точки зрения захоронения высокорадиоактивных отходов атомной промьшленности. Применение технологии самораспространяющегося высокотемпературного синтеза для получения таких материалов является более ресурсосберегающим и не требует сложного технологического оборудования и проведения комплексных операций по сравнению с традиционными способами получения.

Цель: определить и предложить способ получения алюмината неодима в качестве матричного материала актиноидной фрракции радиоактивных отходов.
\end{abstract}

Объекты: алюминат неодима $\mathrm{NdAlO}_{3}$ co структурой минерала перовскит; неодим, являющийся имитатором трёхвалентной фракции радиоактивных отходов.

Методы: оценка и анализ получения матричного материала путем компьютерного моделирования процесса самораспространяющегося высокотемпературного синтеза; проведение лабораторных экспериментов по синтезу алюмината неодима; определение фазового состава разрабатьваемого материала методом рентгенофразового анализа.

Результаты. Описана расчетно-теоретическая модель определения принципиальной возможности процесса самораспространяющегося высокотемпературного синтеза матричного материала на основе алюмината неодима, предназначенного для иммобилизации высокоактивных радиоактивных отходов. Методы численного моделирования позволили рассмотреть различные реакции синтеза такого материала и определить максимально возможное количество включения имитатора трехвалентных актиноидов. Так, для получения $\mathrm{NdAlO}_{3}$ необходимо использовать СBC-реакцию образования алюминийникеля с дополнительным включением не более 46 мас. \% системы $\mathrm{Nd}_{2} \mathrm{O}_{3}-\mathrm{Al}_{2} \mathrm{O}_{3}$ (соотношение компонентов оксидов 3,3:1) в исходную шихту смеси. Базируясь на результатах разработанной модели, было проведено экспериментальное исследование по синтезу матричного материала. Определены оптимальные условия подготовки шихты компонентов: плотность исход-

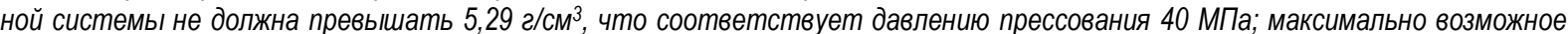
включение $\mathrm{Nd}_{2} \mathrm{O}_{3}-\mathrm{Al}_{2} \mathrm{O}_{3}$ в систему Ni-Al- не более 40 мас. \%. Проведенный рентгенофразовый анализ показал наличие фразы алюмината неодима во всех синтезируемых образцах, максимальная доля $\mathrm{NdAlO} 3$ достигается при синтезе образца с 40 мас. \% добавки $\mathrm{Nd}_{2} \mathrm{O}_{3}-\mathrm{Al}_{2} \mathrm{O}_{3}$ и давлением прессования 30 МПа. Таким образом, использование ресурсоэфффективной и простой технологии самораспространяющегося высокотемпературного синтеза позволяет получить надежный матричный материал для иммобилизации радиоактивных отходов.

Ключевые слова:

Самораспространяющийся высокотемпературный синтез, перовскит, ядерные отходы, иммобилизация, актиноиды.

\section{Введение}

Атомные электростанции, как и любой другой объект энергетики, постоянно генерируют отходы, при этом их количество значительно ниже по сравнению с отходами других видов производства тепловой электроэнергии. Тем не менее активное развитие ядерных программ может привести к существенному накоплению наиболее опасных для человека и всей биосферы в целом высокоактивных радиоактивных отходов (BАО) [1]. Как правило, основным источником таких отходов являются продукты деления и трансурановые элементы, образующиеся в процессе кампании ядерной энергетической установки. Количество ВАО составляет лишь $3 \%$ от общего объема всех отходов атомной энергетики, но в то же время их активность достигает $95 \%$ от суммарной активности
$[2,3]$. Таким образом, необходимость в изоляции наиболее долгоживущих и биологически опасных нуклидов из окружающей среды становится главной задачей обращения с радиоактивными отходами.

В настоящее время одним из наиболее распространенных способов иммобилизации ВАО является применение технологии остекловывания [2-4]. Используемые силикатные и фосфатные стекла представляют собой аморфные материалы с топологически неупорядоченной структурой. Отрицательным аспектом их использования является тот факт, что стекла не могут гарантировать стабильность и надежность консервации актиноидной фракции в течение нескольких тысяч лет в процессе долговременного хранения вследствие их химической нестабильности и тенденции к спонтанной кристаллизации при повышении температур. 
Альтернативными материалами для иммобилизации являются кристаллические матрицы - синтетические аналоги природных геологических минералов. Такие соединения (перовскит, монацит, цирконолит, пирохлор и др.) способны длительное время надежно содержать высокоактивные фракции радиоактивных отходов $[5,6]$. Тем не менее данные материалы не получили широкого применения в связи с отсутствием промышленной технологии синтеза, а существующие методы, такие как индукционная плавка, плазменное и микроволновое плавление, сопровождаются сложными технологическими процессами получения и высокими энергетическими затратами.

Наилучшей технологией изготовления иммобилизационных матриц может являться самораспространяющийся высокотемпературный синтез (СВС). Преимуществами СВС являются возможность получения материалов с заданными свойствами, высокая чистота конечного продукта, низкое энергопотребление и возможность контролировать все стадии процесса синтеза [7-9].

Таким образом, целью данной работы является получение матричного материала перовскитного типа методом самораспространяющегося высокотемпературного синтеза.

\section{Постановка задачи}

Реакция получения алюмината неодима протекает по следующему уравнению:

$$
\mathrm{Nd}_{2} \mathrm{O}_{3}+\mathrm{Al}_{2} \mathrm{O}_{3}=2 \mathrm{NdAlO}_{3} \text {. }
$$

В данном случае $\mathrm{Nd}$ является имитатором трехвалентной актиноидной фракции (Pu, $\mathrm{Am}, \mathrm{Cm}$, и др.) радиоактивных отходов вследствие близости ионных радиусов и, как следствие, схожести химических свойств неодима и актиноидов.

Объединенным количественным критерием принципиальной осуществимости процесса получения химического соединения, позволяющего выяснить степень протекания и трансформации начальных реагентов реакции в конечный продукт, является энергией Гиббса $\Delta \mathrm{G}^{\circ}$. Определение значения стандартной энергии Гиббса реакции (1) базировалось на полуэмпирическом подходе, предложенном О. Фабричной и Г. Зайферта $[10,11]$, а также С. Хуангом и О. Ван дер Бистом [12] (табл. 1). Такой метод показывает достаточно хорошее согласие между экспериментальными и расчетными данными.

Таблица 1. Фазовое описание системы $\mathrm{Nd}_{2} \mathrm{O}_{3}-\mathrm{Al}_{2} \mathrm{O}_{3}$

Table 1. Phase description of the $\mathrm{Nd}_{2} \mathrm{O}_{3}-\mathrm{Al}_{2} \mathrm{O}_{3}$ system

\begin{tabular}{c|c|c}
\hline Фаза/Phase & $\begin{array}{c}\text { Подрешеточная модель } \\
\text { Sublattice model }\end{array}$ & $\begin{array}{c}\text { Полуэмпирическая формула расчета } \Delta \mathrm{G}^{\circ} \\
\text { Semi-empirical formula for calculation } \Delta \mathrm{G}^{\circ}\end{array}$ \\
\hline $\mathrm{Nd}_{2} \mathrm{O}_{3}$ & $\left(\mathrm{Nd}^{+3}\right)_{2}\left(\mathrm{O}^{-2}\right)_{3}$ & $\Delta \mathrm{G}^{\circ}\left(\mathrm{Nd}_{2} \mathrm{O}_{3}\right)=-1847329+637,424 \cdot T-116,358 \cdot T \cdot \ln (T)$ \\
\hline & & $298,14>T>600:$ \\
& & $\Delta \mathrm{G}^{\circ}\left(\mathrm{Al}_{2} \mathrm{O}_{3}\right)=-1707351+448,021 \cdot T-67,4804 \cdot T \cdot \ln (T)$ \\
& & $-0,06747 \cdot T^{2}+1,4205433 \cdot 10^{-5 T^{2}} \cdot+938780 / T$ \\
$\mathrm{Al}_{2} \mathrm{O}_{3}$ & $\left(\mathrm{Al}^{+3}\right)_{2}\left(\mathrm{O}^{-2}\right)_{3}$ & $600>T>1500:$ \\
& & $\Delta \mathrm{G}^{\circ}\left(\mathrm{Al}_{2} \mathrm{O}_{3}\right)=-1724886+754,856 \cdot T-116,258 \cdot \mathrm{T} \cdot \ln (T)$ \\
& & $-0,0072257 \cdot T^{2}+2,78532 \cdot 10^{-7 T^{3}}+2120700 / T$ \\
& & $1500>T>3000:$ \\
& & $\Delta \mathrm{G}^{\circ}\left(\mathrm{Al}_{2} \mathrm{O}_{3}\right)=-1772163+1053,454 \cdot T-156,058 \cdot \mathrm{T} \cdot \ln (T)+0,007091 \cdot T^{2}-6,29402 \cdot 10^{-7 T^{3}}+12366650 / T$ \\
\hline $\mathrm{NdAlO}_{3}$ & $\left(\mathrm{Nd}^{+3}\right)\left(\mathrm{Al}^{-2}\right)\left(\mathrm{O}^{-2}\right)_{3}$ & $\Delta \mathrm{G}^{\circ}\left(\mathrm{NdAlO}_{3}\right)=0,5 \cdot \Delta \mathrm{G}^{\circ}\left(\mathrm{Nd}_{2} \mathrm{O}_{3}\right)+0,5 \cdot \Delta \mathrm{G}_{\text {corund }}^{\circ}-41197-15,794 \cdot T$
\end{tabular}

Графическая зависимость энергии Гиббса от температуры, в соответствии с описанными выше полуэмпирическими выражениями, для системы $\mathrm{Nd}_{2} \mathrm{O}_{3}$ $\mathrm{Al}_{2} \mathrm{O}_{3}$ представлена на рис. 1 .

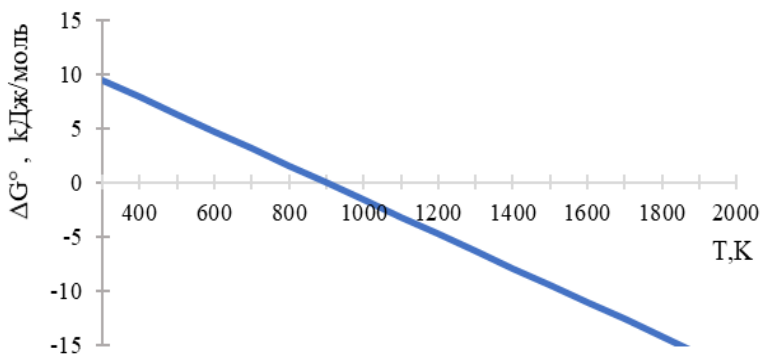

Pис. 1. Зависимость энергии Гиббса образования алюмината неодима, рассчитанной в соответствии с полуэмпирическими зависимостями

Fig.1. Dependence of Gibbs energies of the neodymium aluminate formation calculated by semi-empirical formulas

Как видно из графика, реакция получения алюмината неодима возможна лишь при температуре около
900 К и выше, что достаточно точно согласуется с экспериментальными данными ряда работ по синтезу $\mathrm{NdAlO}_{3}[13]$.

\section{Расчетно-теоретическая модель получения алюмината неодима}

Для осуществления процесса синтеза методом СВС необходимо, чтобы рассматриваемая реакция была экзотермической, так как только для данного рода реакций наблюдается процесс распространения фронта горения в автоволновом режиме, при котором выделяющееся тепло передается от слоя к слою путем теплопередачи. Следовательно, необходимо использование дополнительной СВС-реакции с большим энергетическим выходом, при которой часть тепла будет поглощаться системой $\mathrm{Nd}_{2} \mathrm{O}_{3}-\mathrm{Al}_{2} \mathrm{O}_{3}$ для необходимого разогрева исходных оксидов и последующего образования алюмината неодима.

Выбор композиции должен определяться не только энергетическим выходом реакции синтеза, но и комплексом свойств образующегося соединения, обеспечивающего практическую полезность его применения в качестве матричного каркаса. Так, напри- 
мер, многие силициды имеют высокую жаростойкость, бориды повышают стойкость к износу, станниды обладают сверхпроводящими свойствами и т. д.

Другими критериями выбора являются минимизация количества образующихся фазовых составляющих продуктов и наличие температур плавления, существенно ниже развивающихся в процессе синтеза температур. Плавление фазовых компонентов позволяет достигать появления жидкостных фаз в структуре продуктов, а затем, в процессе остывания и затвердевания, создания дополнительного упрочняющего каркаса. Таким образом, синтезируется матричный материал, представляющий собой блок с диспергированными фазовыми составляющими

Кроме того, температура синтеза не должна превышать температуру плавления алюмината неодима (2489 K) более чем на 100 К, так как при дальнейшем повышении температуры возможно его разложение [14]. Количество компонентов и их элементарный состав не должны оказывать сильного эффекта на гидролитические свойства матрицы на основе алюмината неодима.

Наиболее распространенные СВС-реакции с большим энергетическим выходом и подходящие к вышеописанным критериям представлены в табл. 2.

Таблица 2. СВС-реакции с высоким энергетическим выходом $[15,16]$

Table 2. SHS-reactions with high-energy yield [15, 16$]$

\begin{tabular}{|c|c|}
\hline Реакция/Reaction & $\mathrm{Q}$, кДж/г $(\mathrm{\kappa J} / \mathrm{g})$ \\
\hline $\mathrm{Ti}+2 \mathrm{~B}=\mathrm{TiB}_{2}$ & 4,02 \\
\hline $\mathrm{V}+2 \mathrm{~B}=\mathrm{VB}_{2}$ & 2,81 \\
\hline $\mathrm{Ti}+\mathrm{C}=\mathrm{TiC}$ & 3,08 \\
\hline $\mathrm{Ti}+2 \mathrm{Si}=\mathrm{TiSi}_{2}$ & 1,31 \\
\hline $\mathrm{Zr}+\mathrm{N}=\mathrm{ZrN}$ & 3,41 \\
\hline $\mathrm{Ni}+\mathrm{Al}=\mathrm{NiAl}$ & 1,37 \\
\hline
\end{tabular}

Диборид титана обладает исключительной твердостью, устойчив в водных и кислотных растворах, в процессе окисления на его поверхности образуется стекловидная пленка, являющаяся дополнительным барьером к процессам выщелачивания $[17,18] . \mathrm{VB}_{2}$, как и всем боридам пятой группы, присущи высокая химическая и гидролитическая стойкость, твердость, механическая прочность [19]. Высокие температуры плавления и теплота образования карбида титана (3260 ${ }^{\circ} \mathrm{C}$ и 184,76 кДж/моль соответственно [20]) служат доказательством его стабильности. TiC, как и $\mathrm{TiSi}_{2}$, устойчивы к агрессивным средам, коррозионностойки, что обеспечивает их широкое применение в различных областях науки и техники [21]. Нитрид циркония - материал с высокой твердостью, имеет высокую устойчивость к процессам выщелачивания и износу [22]. $\mathrm{NiAl}$ обладает низкой плотностью, высокой температурой плавления, хорошей стойкостью к окислению и коррозии. При синтезе $\mathrm{NiAl}$ возможно получение дополнительных фаз - $\mathrm{Ni}_{2} \mathrm{Al}_{3}, \mathrm{NiAl}_{3}, \mathrm{Ni}_{3} \mathrm{Al}$, значительно повышающих его прочностные характеристики вследствие искажения кристаллической решетки [20].

Таким образом, предложенные соединения, с одной стороны, являются конечными продуктами СВСреакций с необходимым энергетическим выходом, направленным на образования $\mathrm{NdAlO}_{3}$, а с другой стороны, могут способствовать улучшению характеристик синтезируемой матрицы.

Особое влияние при реализации реакций самораспространяющегося высокотемпературного синтеза имеет связь максимальной температуры процесса горения с начальной температурой системы и количеством включения в нее различных продуктов. Так, например, при превышении некоторого предельного значения содержания добавки реакция синтеза может вообще не реализоваться или «погаснуть» в процессе синтеза. Следовательно, при исследовании применимости реакций, представленных в таблице, необходимо учитывать также и критерии осуществления CBC-реакций.

Как правило, существующие модели, характеризующие кинетику процесса самораспространяющегося высокотемпературного синтеза, описывают довольно элементарные одностадийные случаи. Невозможность воссоздания поведения всех протекающих реакций в волне горения в единице объема реальных систем накладывает существенные ограничения при анализе процесса СBC.

Недостаточность расчетных данных, характерная для создания новых перспективных материалов с заданными свойствами, синтезируемыми в случае реализации многостадийных реакций, также является дополнительным лимитирующим фактором определения параметров твердофазного горения.

Выходом из создавшегося положения является проведение термодинамического анализа, в частности предварительный термодинамический расчет адиабатической температуры горения $-T_{\text {ад}}$.

Так, результаты экспериментальных исследований СВС-процессов показали, что при:

- $T_{\text {ад }}<1500 \mathrm{~K}$ горение не осуществимо для любых систем;

- $T_{\text {ад }}>2000 \mathrm{~K}$ системы всегда горят;

- $1500 \mathrm{~K}<T_{\text {ад }}<2000 \mathrm{~K}$ горение может как осуществляться, так и не осуществляться, необходимы дополнительные исследования [23].

Пусть в результате самораспространяющегося высокотемпературного синтеза образуются полностью прореагировавшие продукты реакции и энергия реакции в виде выделяющегося тепла расходуется на изменение начальной температуры до значения температуры адиабатической. Из равенства энтальпий продуктов реакции при адиабатической температуре $T_{\text {ад }}$ и начальных реагентов при температуре инициирования $T_{0}$ следует [24], что:

$$
\sum_{i=1}^{n}\left[H\left(T_{\text {ад }}\right)-H\left(T_{0}\right)\right]=Q,
$$

где $H\left(T_{\text {ад }}\right)$ и $H\left(T_{0}\right)$ - энтальпии продуктов при адиабатической и начальной температурах; $Q$ - теплота реакции, выделяемая в процессе синтеза. Суммирование следует проводить по всем образующимся в реакции продуктам. Величина энергетического эффекта рассчитывается в соответствии со значениями стандартных энтальпий образования. 
Рассмотрим случай реализации прохождения реакции при образовании двух конечных продуктов в соответствии со следующей схемой:

$$
\sum_{j-1}^{m} X_{j} \rightarrow Z_{1}+Z_{2}
$$

где $X_{1}$ - исходный компонент $j$-го сорта; $Z_{1}$ - продукт

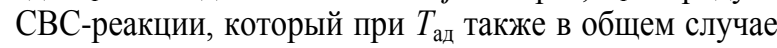
может находиться как в твердой, так и в жидкой фазе; $Z_{2}$ - продукт, полученный в результате включения дополнительных реагентов в исходную систему.

Исходя из того, что исходные компоненты взаимодействуют полностью, соотношение между продуктами можно определить в соответствии со стехиометрией уравнения.

Для представленной схемы осуществления процесса синтеза исходное уравнение (2) принимает вид:

$$
\int_{T_{0}}^{T_{\text {ag }}} c(T) d T=(1-x)(Q-v M)-x L,
$$

где $c(T)$ - температурная зависимость теплоемкости продукта реакции $\mathrm{CBC} ; L$ - величина теплового эффекта образования продукта $Z_{2}$ из компонентовразбавителей; $x$ - доля содержания дополнительных продуктов-компонентов в исходной шихте (массовая, объемная или мольная в зависимости от используемых единиц измерения величин $c(T), Q, M) ; M$ - теплота плавления продукта $Z_{1} ; v$ - доля жидкой фазы в конечном продукте $Z_{1}$, причем:

$$
\begin{aligned}
& v=0 \text { если } T_{\text {пл }}>T_{\text {ад }} ; \\
& v=1 \text { если } T_{\text {пл }}<T_{\text {ад }},
\end{aligned}
$$

Для выявления влияния процесса плавления первой компоненты $Z_{1}$ на протекание СВС необходимо определить взаимосвязь адиабатической температуры с температурой плавления и рассчитать параметр [24]:

$$
\Delta H\left(T_{\text {пл }}\right)=\int_{T_{0}}^{T_{\text {пा }}} c(T) d T,
$$

и сравнить ее с величинами $Q$ и $Q-M$.

В случае, если:

- $\Delta H\left(T_{\text {пл }}\right)>Q$, то $T_{\text {пл }}>T_{\text {ад }}$;

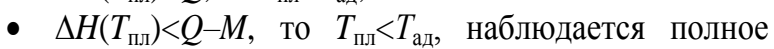
плавление продукта.

Если же $Q-M<\Delta H\left(T_{\text {пл}}\right)<Q$, то $T_{\text {пл}}=T_{\text {ад, }}$ происходит частичное плавление компонента, коэффициент $v$ необходимо находить из следующего выражения:

$$
v=\frac{Q-\Delta H\left(T_{\text {пл }}\right)}{M} .
$$

Решение уравнения (3) позволяет определить зависимость адиабатической температуры $T_{\text {ад }}$ от параметров подготовки исходной шихты, т. е. степени содержания добавки в исходной системе $x$ и температуры инициирования реакции $T_{0}$, а также проанализировать вероятность осуществления процесса горения в системах с образованием двух продуктов реакции в соответствии с вышеописанными численными критериями.

\section{Экспериментальное получение алюмината неодима}

Синтез матричных материалов методом СВС осуществлялся по следующей схеме (рис. 2).

где $T_{\text {пл }}-$ температура плавления продукта.

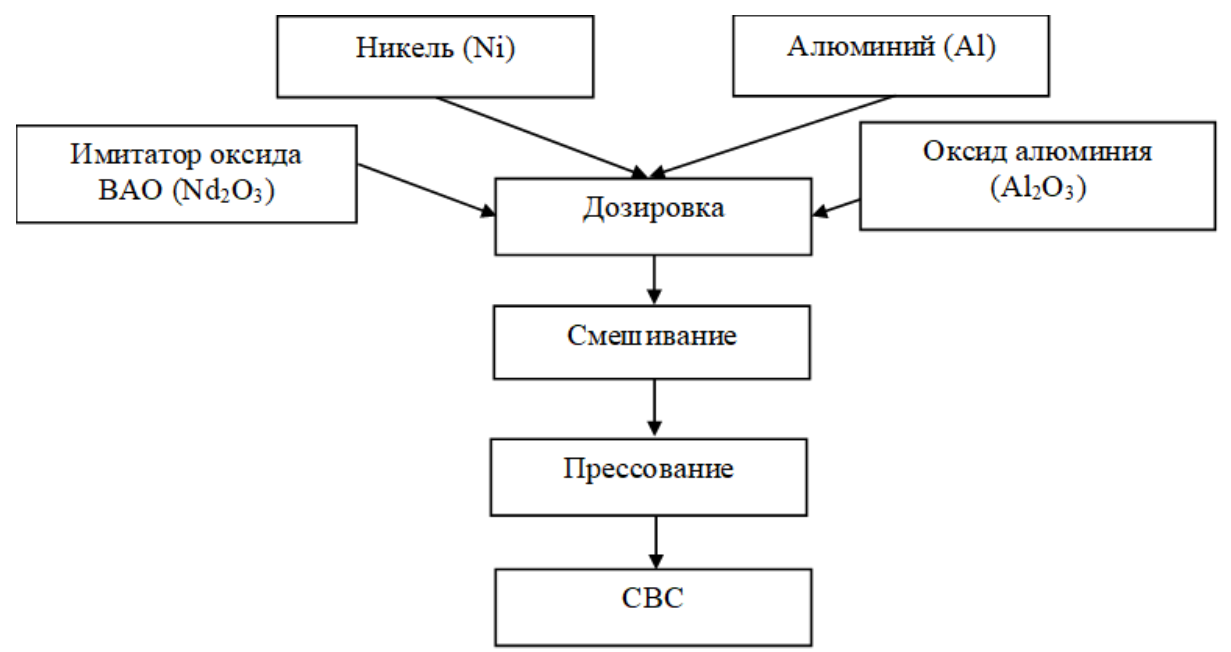

Рис. 2. Схема получения матричного материала

Fig. 2. Scheme of matrix material production

Подготовка шихты для проведения процесса синтеза осуществлялась следующим образом. На первом этапе исходные реагенты: оксиды алюминия и актиноида (неодима), промышленно изготовленные порошки алюминия и никеля, дозировались весовым методом из расчета осуществления процесса СBC $\mathrm{NiAl}$ с образованием алюмината неодима: $\mathrm{Al}$ и $\mathrm{Ni}-$
31,5 и 68,5 масс. \% соответственно; к ним добавляется $x$ масс. \% добавки оксидов актиноида и алюминия. При этом дозировка оксидов осуществлялась в соотношении: 3,3:1. На следующем этапе полученная смесь помещалась в шнековый смеситель штырькового типа AR 403 All-Purpose Equpiment (Германия) с насадкой из металлического вращающегося куба для 
осуществления равномерного перемешивания. По истечении 30 минут полученная шихта извлекалась и помещалась в цилиндрическую пресс-форму диаметром 25 мм. Прессование выполнялось с помощью гидравлического лабораторного пресса ПГЛ-12 (максимальное давление 50 МПа на поверхность площадью $30 \mathrm{~cm}^{2}$ ) с целью получения образцов разной плотности при вариации давления от 10 до 40 МПа с шагом в 10 МПа. Время выдержки составляло 1 час. На следующем этапе шихта извлекалась из прессформ и помещалась в СВС-реактор для прогрева образцов и осуществления СВС. Детектирование температур осуществлялось с помощью вольфрамрениевых термопар, подключенных к многоканальному счетчику импульсов Овен СИ8. Синтез осуществлялся в условиях остаточного давления 300 Па.

Фазовый анализ полученных образцов матричного материала осуществлялся на рентгеновском дифрактометре Shimadzu XRD 6000 с использованием CuKaизлучения.

\section{Результаты и обсуждение}

Рассмотрим реакцию образовании алюмината неодима с помощью CBC-реакции получения $\mathrm{NiAl}$.
Для определения расчетно-теоретической возможности получения композиционных материалов на основе алюмината неодима в режиме технологического горения исходные реагенты смешиваются в весовых соотношениях в соответствии с осуществлением следующей реакции:

$$
\mathrm{Al}+\mathrm{Ni}+x\left(\mathrm{Nd}_{2} \mathrm{O}_{3}+\mathrm{Al}_{2} \mathrm{O}_{3}\right)=2 \mathrm{NdAlO}_{3}+\mathrm{NiAl} .
$$

Так как расчет предполагает, что потери массы системой в процессе синтеза отсутствуют, то можно утверждать, что сохранность массовых соотношений между составляющими сложной системы до и после синтеза соблюдается. Массовая доля $x$-содержание компонентами оксидов неодима и алюминия в весовом соотношении 3,3:1 в исходной шихте CBCреакции.

При реализации численных экспериментов рассматривалась зависимость адиабатической температуры горения данной системы от содержания количества включения $x$ системы $\mathrm{Nd}_{2} \mathrm{O}_{3}-\mathrm{Al}_{2} \mathrm{O}_{3}$ в исходную шихту компонентов (рис. 3). На рис. 4 показано влияние температуры предварительного подогрева на развитие адиабатической температуры в процессе синтеза, рассчитанная в соответствии с уравнением (4).

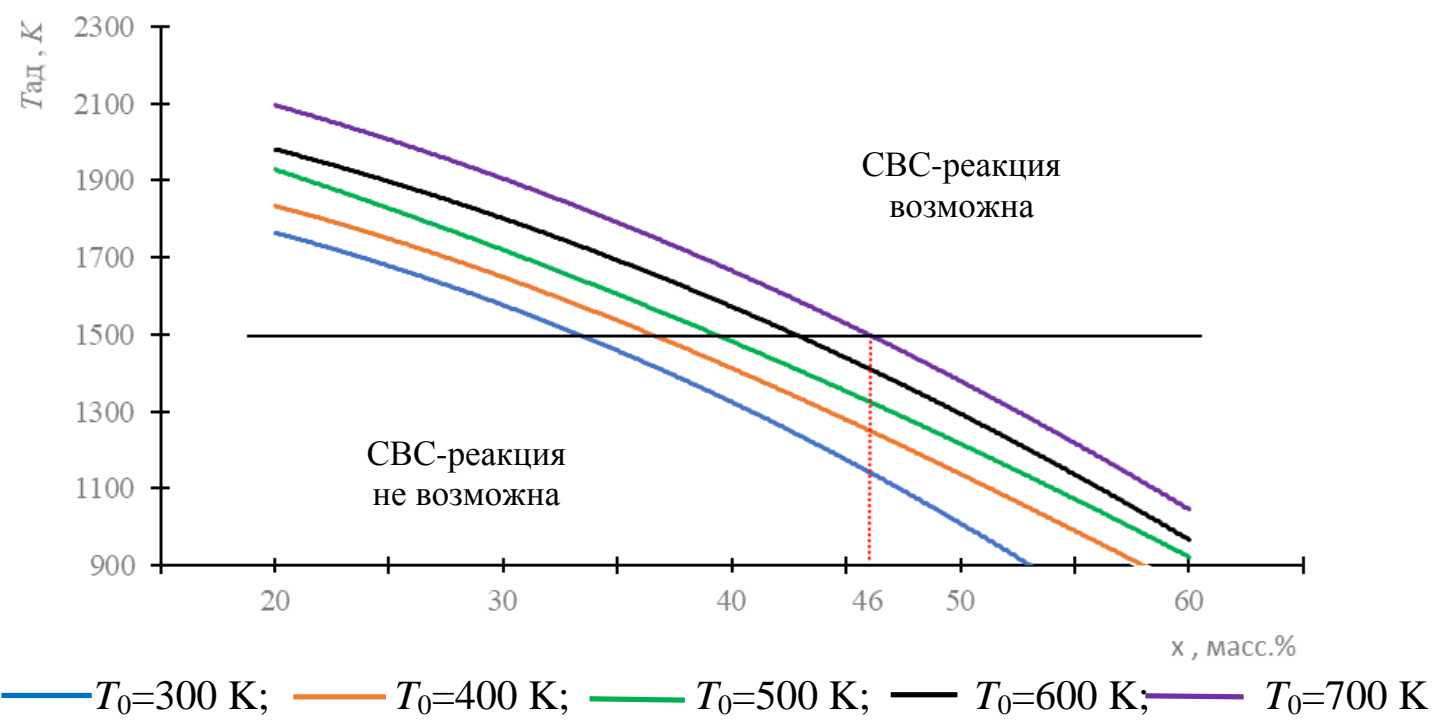

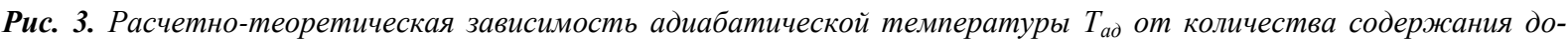
бавки $\mathrm{Nd}_{2} \mathrm{O}_{3}-\mathrm{Al}_{2} \mathrm{O}_{3} x$ в исходной шихте при осуществлении $\mathrm{CBC}$-реакции образования $\mathrm{NiAl}$ и варьировании температуры предварительного подогрева $T_{0}$

Fig. 3. Calculated theoretical dependence of adiabatic temperature $T_{a d}$ on amount of additive content $\mathrm{Nd}_{2} \mathrm{O}_{3}-\mathrm{Al}_{2} \mathrm{O}_{3} \times$ for $S H S$-reaction of NiAl during varying the preheating temperature $T_{0}$

Анализ расчетных зависимостей $T_{\text {ад }}$ от перечисленных выше параметров показал, что для системы, включающей долю компоненты $\mathrm{Nd}_{2} \mathrm{O}_{3}-\mathrm{Al}_{2} \mathrm{O}_{3}$ выше 46 мас. \% $(x=46 \%)$, горение не реализуется при всех вариациях изменяемых параметров (температуре предварительного подогрева 700 К). При исследовании влияния температуры предварительного подогрева на изменение $T_{\text {ад }}$ было установлено, что процесс горения возможен без предварительного подогрева, но с количеством добавки не более 34 мас. $\%\left(T_{0}=300 \mathrm{~K}\right)$.
Аналогичным методом был произведен термодинамический расчет адиабатической температуры для реакций, описанных в табл. 3.

При реализации реакций синтеза $\mathrm{TiB}_{2}, \mathrm{TiC}$ и $\mathrm{ZrN}$ методом СВС развиваемые адиабатические температуры значительно выше порогового значения температуры ( 2600 K), при которой возможно разложение алюмината неодима на составляющие оксиды.

$\mathrm{CBC} \mathrm{VB}_{2}$ (рис. 5) с включением в исходную систему около 46 мас. \% $\mathrm{Nd}_{2} \mathrm{O}_{3}-\mathrm{Al}_{2} \mathrm{O}_{3}$ требует достижения высоких температур подогрева (выше $1100 \mathrm{~K}$ ) и значительных энергозатрат. Снижение температуры 
до 800 К позволит надежно иммобилизировать не бо- лее 39 мас. \% добавки.

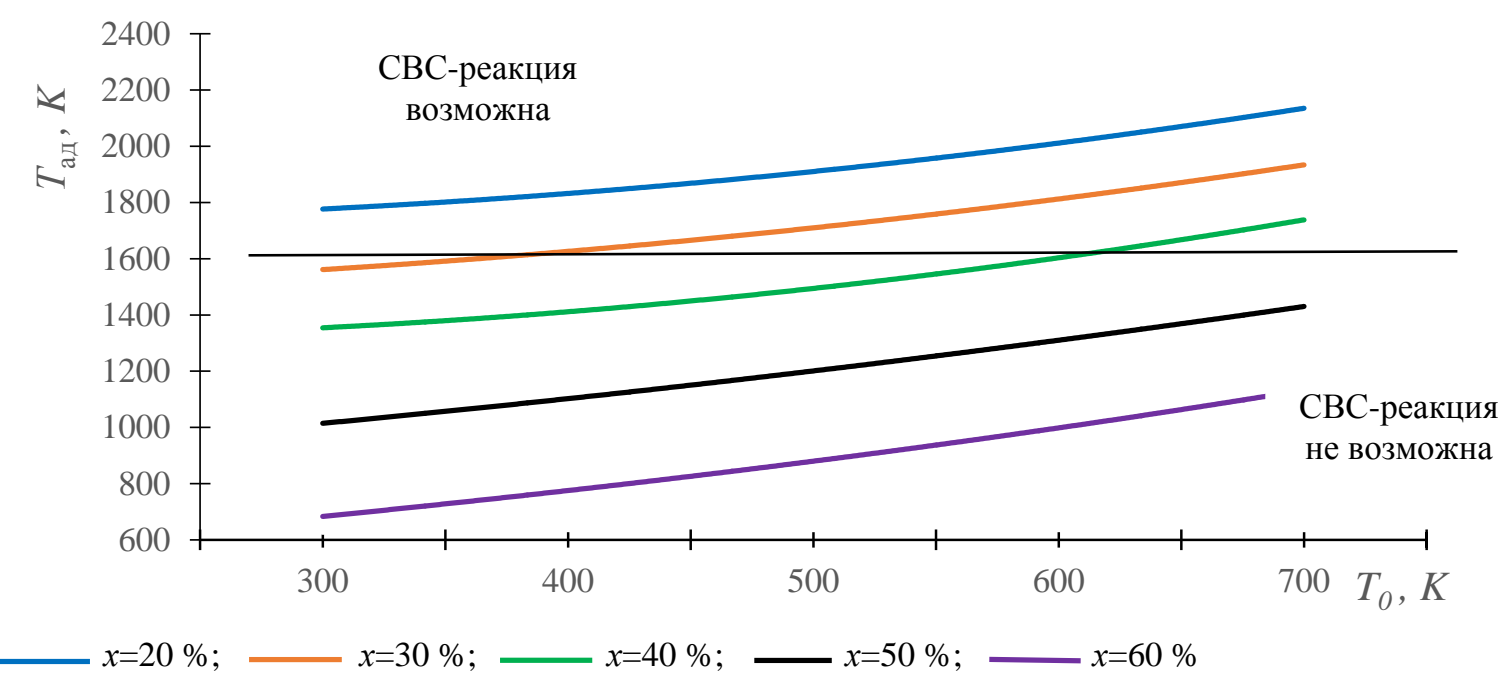

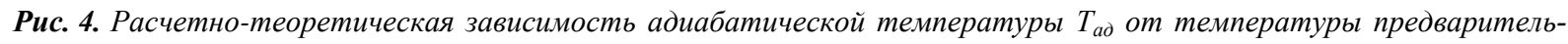
ного подогрева $T_{0}$ для СBC реакции образования $\mathrm{NiAl}$ и варьировании количества содержания добавки $\mathrm{Nd}_{2} \mathrm{O}_{3^{-}}$ $\mathrm{Al}_{2} \mathrm{O}_{3}$ х в исходной иихте

Fig. 4. Calculated theoretical dependence of adiabatic temperature $T_{a \partial}$ on the preheating temperature $T_{0}$ for SHS-reaction of $\mathrm{NiAl}$ during varying amount of additive content $\mathrm{Nd}_{2} \mathrm{O}_{3}-\mathrm{Al}_{2} \mathrm{O}_{3} \mathrm{x}$ in the initial mixture

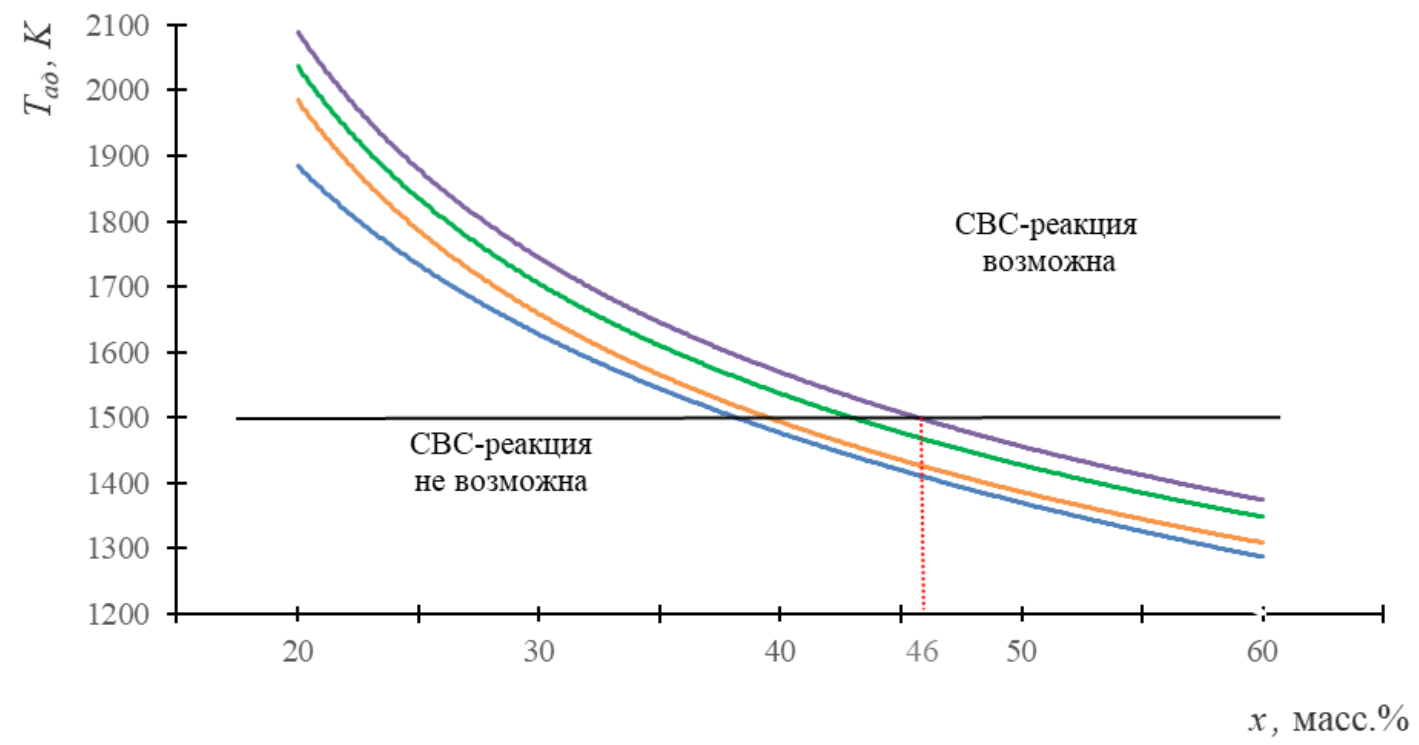

$-800 \mathrm{~K} ; \quad-1000 \mathrm{~K} ; \quad-1100 \mathrm{~K}$

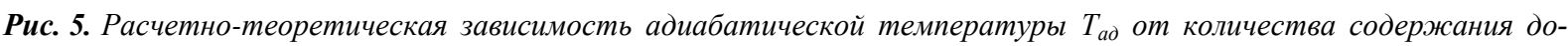
бавки $\mathrm{Nd}_{2} \mathrm{O}_{3}-\mathrm{Al}_{2} \mathrm{O}_{3}$ х для CВC-реакции образования $\mathrm{VB}_{2}$ и варьировании температуры предварительного подогрева $T_{0}$

Fig. 5. Calculated theoretical dependence of adiabatic temperature $T_{a \partial}$ on amount of additive content $\mathrm{Nd}_{2} \mathrm{O}_{3}-\mathrm{Al}_{2} \mathrm{O}_{3} \times$ for $S H S$-reaction of $\mathrm{VB}_{2}$ during varying the preheating temperature $T_{0}$

Максимально возможное включение добавки при синтезе $\mathrm{TiSi}_{2}$ (рис. 6) составляет не более 42 мас. \% при температуре подогрева около 700 К. Для реакции синтеза NiAl предельное включение $\mathrm{Nd}_{2} \mathrm{O}_{3}-\mathrm{Al}_{2} \mathrm{O}_{3}$ равно 46 мас. \% при температуре предварительного подогрева $700 \mathrm{~K}$.

Из этого следует, что использование реакции синтеза NiAl в режиме технологического горения для получения матричного материала, содержащего алюминат неодима, является наиболее приемлемым среди рассмотренных.
Лабораторные эксперименты по синтезу матричного материала на основе алюмината неодима осуществлялись в соответствии с вышеописанной методикой.

По результатам численного моделирования была проведена серия экспериментов (табл. 3) по получению матричного материала с целью исследования влияния плотности шихты компонентов и степени включения имитатора отходов $\mathrm{Nd}_{2} \mathrm{O}_{3}$ на процесс синтеза и фазовый состав конечного продукта. Все об- 
разцы изготавливались в количестве трех единиц для $\quad$ набора статистики проведения экспериментов.

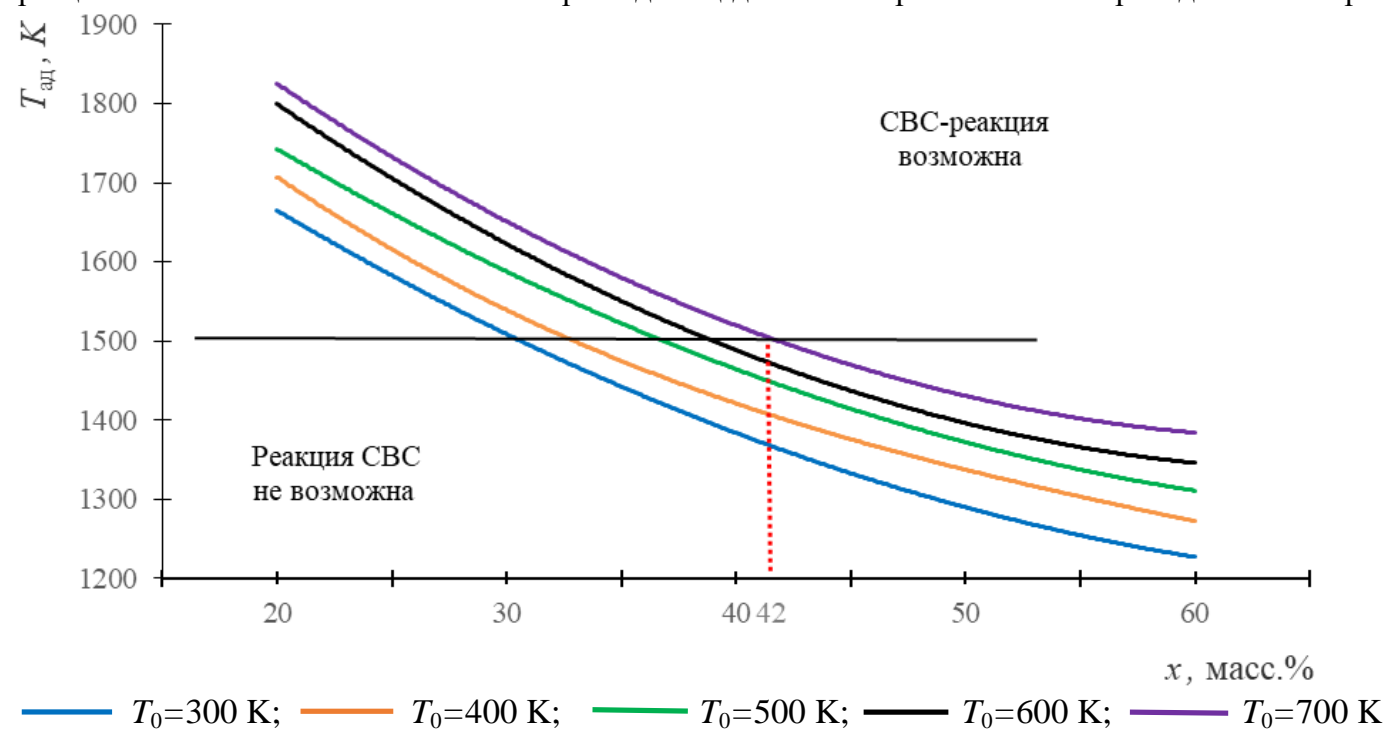

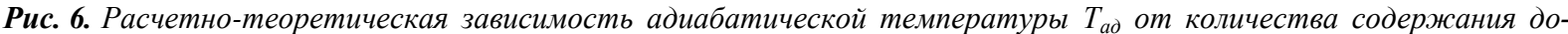
бавки $\mathrm{Nd}_{2} \mathrm{O}_{3}-\mathrm{Al}_{2} \mathrm{O}_{3}$ хдля CВС-реакиии образования $\mathrm{TiSi}_{2}$ и варьировании температуры предварительного подогрева $T_{0}$

Fig. 6. Calculated theoretical dependence of adiabatic temperature $T_{a \partial}$ on amount of additive content $\mathrm{Nd}_{2} \mathrm{O}_{3}-\mathrm{Al}_{2} \mathrm{O}_{3} \times$ for $\mathrm{SHS}$-reaction of $\mathrm{TiSi}_{2}$ during varying the preheating temperature $T_{0}$

Таблица 3. Параметры подготовки иихты компонентов перед проведением СВС

Table 3. Preparation parameters of batch before carrying out SHS

\begin{tabular}{|c|c|c|c|c|c|c|c|c|c|c|c|c|}
\hline $\begin{array}{l}\text { Номер образца } \\
\text { Sample number }\end{array}$ & 1 & 2 & 3 & 4 & 5 & 6 & 7 & 8 & 9 & 10 & 11 & 12 \\
\hline $\begin{array}{l}\text { Давление прессования Р, МПа } \\
\text { Pressure P, MPa }\end{array}$ & \multicolumn{3}{|c|}{10} & \multicolumn{3}{|c|}{20} & \multicolumn{3}{|c|}{30} & \multicolumn{3}{|c|}{40} \\
\hline $\begin{array}{l}\text { Среднее значение плотности об- } \\
\text { разцов } \rho, \Gamma / \mathrm{cm}^{3} \\
\text { Average value of sample density } \rho, \mathrm{g} / \mathrm{cm}^{3}\end{array}$ & 4,86 & 4,90 & 4,93 & 5,07 & 5,04 & 5,02 & 5,18 & 5,16 & 5,14 & 5,29 & 5,33 & 5,32 \\
\hline $\begin{array}{l}\text { Включение добавки } \mathrm{Nd}_{2} \mathrm{O}_{3}-\mathrm{Al}_{2} \mathrm{O}_{3} \text {, } \\
\text { мac. \% } \\
\text { Inclusion of } \mathrm{Nd}_{2} \mathrm{O}_{3}-\mathrm{Al}_{2} \mathrm{O}_{3} \text { additive, } \\
\text { wt. \% }\end{array}$ & 30 & 40 & 50 & 30 & 40 & 50 & 30 & 40 & 50 & 30 & 40 & 50 \\
\hline $\begin{array}{l}\text { Характер волны горения в процес- } \\
\text { ce CBC* } \\
\text { Behavior of the combustion wave } \\
\text { during SHS* }\end{array}$ & + & + & - & + & + & - & + & + & - & $\square$ & $\square$ & - \\
\hline
\end{tabular}

*Примечание: «+»-устойчивое распространение волны горения; «-»-затухание волны горения в процессе синтеза; «" - нестационарное поведение волны горения с последующим термомеханическим разрушением образцов.

*Note: «+»- stable propagation of the combustion wave; «-»-attenuation of the combustion wave during synthesis, « $\square »-$ unstable behavior of the combustion wave with subsequent thermomechanical destruction of the samples.

В результате проведения СВС было установлено, что при нагреве исходных образцов № 3, 6, 9, 12 наблюдаются локальные очаги горения без разрастания в волну с последующим затуханием реакции синтеза вне зависимости от плотности шихты, что обусловлено излишним содержанием добавки и повышенными энергетическим затратами на осуществление реакции синтеза алюмината неодима.

При синтезе образцов № 10, 11 наблюдается термомеханическое разрушение и нестационарность процесса распространения волны горения вследствие избыточной плотности исходной шихты и резкого удельного энергетического выхода реакций, протекающих в единице объёма образца.

В процессе нагрева образцов № 1, 2, 4, 5, 7, 8 до температур 650-750 К (вследствие различных харак- теристик начальной шихты компонентов реакции) происходит инициирование волны горения на торце с последующим развитием по всему объему образцу, наблюдается стремительный скачок температуры со стабилизацией в районе $1600-1950 \mathrm{~K}$, то есть процесс синтеза протекает практически в изотермическом режиме. В дальнейшем образцы остывали до температуры окружающей среды.

В соответствии с полученными данными наблюдается достаточная согласованность между результатами численного и лабораторного экспериментов по определению влияния содержания добавки $\mathrm{Nd}_{2} \mathrm{O}_{3}-\mathrm{Al}_{2} \mathrm{O}_{3}$ в системе $\mathrm{Ni}$-Al на синтез матричного материала на основе алюмината неодима (отклонение составляет не более $6 \%$ ). 
Результаты рентгенофазового анализа полученных образцов представлены в табл. 4. Все синтезируемые образцы вне зависимости от условий подготовки исходной шихты включают в себя наличие фазы алюмината неодима $\mathrm{NdAlO}_{3}$ от 16,7 до 39,5 мас. \%. Максимальная доля перовскита достигается при синтезе образца с 40 мас. \% добавки $\mathrm{Nd}_{2} \mathrm{O}_{3}-\mathrm{Al}_{2} \mathrm{O}_{3}$ и давлением прессования 30 МПа.

Таблица 4. Результаты РФА синтезированных образцов Table 4. XRD results of the synthesized samples

\begin{tabular}{|c|c|c|c|c|c|c|c|c|}
\hline \multirow{2}{*}{ 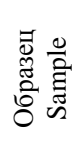 } & \multirow[b]{2}{*}{$\begin{array}{c}\mathrm{P}, \mathrm{MПа} \\
(\mathrm{MPa})\end{array}$} & \multirow[b]{2}{*}{$\begin{array}{c}x \text {, мас. \% } \\
\text { (wt. \%) }\end{array}$} & \multicolumn{6}{|c|}{$\begin{array}{l}\text { Фазовый состав, мас. \% } \\
\text { Phase composition, wt. \% }\end{array}$} \\
\hline & & & $\underset{\overbrace{}}{\stackrel{\infty}{Z}}$ & 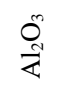 & $\begin{array}{l}\underset{J}{Z} \\
\dot{Z}\end{array}$ & $\underset{z}{\sum_{z}}$ & 苔 & 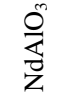 \\
\hline 1 & 20 & 20 & 2,2 & 7,3 & 33,3 & 17,1 & 23,3 & 16,7 \\
\hline 2 & 30 & 20 & 2,5 & 4,6 & 30,4 & 2,4 & 32,7 & 27,4 \\
\hline 4 & 20 & 30 & 2,9 & 6,5 & 31,8 & 13,8 & 25,3 & 19,7 \\
\hline 5 & 30 & 30 & 4,7 & 3,9 & 26,2 & 1,1 & 28,2 & 35,8 \\
\hline 7 & 20 & 40 & 8,3 & 6,9 & 26,3 & 14,2 & 19,8 & 24,5 \\
\hline 8 & 30 & 40 & 3,8 & 5,7 & 26,8 & - & 24,2 & 39,5 \\
\hline
\end{tabular}

Во всех образцах наблюдаются фазы непрореагировавших реагентов вне зависимости от приложенного давления прессования и плотности в количестве: $\mathrm{Nd}_{2} \mathrm{O}_{3} 2,2-8,3$ масс. \%; $\mathrm{Al}_{2} \mathrm{O}_{3} 3,9-7,3$ масс. \% При увеличении содержания количества разбавителя $\mathrm{Nd}_{2} \mathrm{O}_{3}-\mathrm{Al}_{2} \mathrm{O}_{3}$ в системе исходных реагентов $\mathrm{Ni}-\mathrm{Al}$ доля непрореагировавших продуктов возрастает при одном и том же значении плотности. Тем не менее повышение давления прессования позволяет сместить фазообразование в сторону содержания алюмината неодима вследствие роста площади контакта между частицами реагентов внутри шихты компонентов. Увеличение содержания количества оксидных фракций, то есть повышение содержания разбавителей в

\section{СПИСОК ЛИТЕРАТУРЫ}

1. Zhang J. Nuclear fuel reprocessing and waste management. Singapore: World Scientific Publishing Company, 2018. - 279 p. URL: https://doi.org/10.1142/11017 (дата обращения 15.08.2021)

2. Theodore M.K., Theodore L. Introduction to environmenta management. - Boca Raton: CRC Press, 2021. - 556 p. URL: https://doi.org/10.1201/9781003171126 (дата обращения 15.08.2021).

3. Colburn H.A., Peterson R.A. A history of Hanford tank waste, implications for waste treatment, and disposal // Environmental Progress and Sustainable Energy. - 2021. - V. 40. - 12 p. URL: https://doi.org/10.1002/ep.13567 (дата обращения 15.08.2021).

4. Solberg-Johansen B. Life cycle assessment of the nuclear fuel cycle. - London: Academic Press, 1998. - 349 p.

5. Investigating hollandite-perovskite composite ceramics as a potential waste form for immobilization of radioactive cesium and strontium / J. Ma, Z. Fang, X. Yang, B. Wang, F. Luo, X. Zhao, X. Wang, Y. Yang // Journal of Materials Science. - 2021. - V. 56. P. 9644-9654. URL: https://doi.org/10.1007/s10853-021-05886-2 (дата обращения 15.08.2021).

6. High-entropy $\mathrm{A}_{2} \mathrm{~B}_{2} \mathrm{O}_{7}$-type oxide ceramics: a potential immobilising matrix for high-level radioactive waste / L. Zhoua, F. Lia, J. Liu, S. Sun. Y. Liang, G. Zhang // Journal of Hazardous Materials. - 2021. - V. 415. - 9 p. URL https://doi.org/10.1016/j.jhazmat.2021.125596 (дата обращения 15.08.2021) исходной шихте, приводит к росту фазообразования $\mathrm{Ni}_{2} \mathrm{Al}_{3}$ и $\mathrm{NiAl}_{3}$ вследствие понижения температуры синтеза и увеличения энергопотерь на образование алюмината неодима.

Кроме всего прочего, включение дополнительных фаз алюминий-никеля $\mathrm{Ni}_{2} \mathrm{Al}_{3}$ и $\mathrm{NiAl}_{3}$ с искаженными кристаллическими решетками по сравнению с $\mathrm{NiAl}$ в конечный синтезируемый продукт должно привести к увеличению прочностных свойств матричного материала.

\section{Выводы}

В результате проделанной работы можно сделать следующие выводы:

1. Разработана расчетно-теоретическая модель самораспространяющегося высокотемпературного синтеза матричного материала на основе алюмината неодима с учетом максимально возможного включения актиноидной фракции радиоактивных отходов.

2. Определены основные условия синтеза иммобилизационной матрицы $\mathrm{NdAlO}_{3}$ путем включения компонентов имитатора высокоактивных отходов в исследуемую реакцию получения алюминийникеля: содержание включения в исходную систему оксидов $\mathrm{Nd}_{2} \mathrm{O}_{3}-\mathrm{Al}_{2} \mathrm{O}_{3}$ не должно превышать 46 мас. \% (весовое соотношение 3,3:1) при температуре предварительного подогрева $700 \mathrm{~K}$.

3. Лабораторные эксперименты по получению матричного материала показали наличие фазы алюмината неодима во всех образцах. Максимальная доля перовскита достигается при синтезе образца с 40 мас. \% добавки $\mathrm{Nd}_{2} \mathrm{O}_{3}-\mathrm{Al}_{2} \mathrm{O}_{3}$ (весовое соотношение $3,3: 1$ ) и давлением прессования 30 МПа.

7. Self-propagating high-temperature synthesis of ceramic matrices for immobilization of actinide-containing wastes / T.V. Barinova, K.B. Podbolotov, I.P. Borovinskaya, A.S. Shchukin // Radiochemistry. - 2014. - V. 56. - P. 554-559. URL: https://doi.org/10.1134/S1066362214050178 (дата обращения 15.08.2021).

8. Производство порошковых тугоплавких продуктов самораспространяющегося высокотемпературного синтеза в режиме непрерывного горения / С.М. Гайдар, В.Д. Жигарев, А.А. Волков, М.Ю. Карелина // Технология металлов. 2017. - № 7. - C. 37-41.

9. Activation of the technological combustion process of oxide systems by different modifying additives / Z.A. Mansurov, N.N. Mofa, B. S. Sadykov, T.A. Shabanova // Ceramic Engineering and Science Proceedings. - 2013. - V. 2. - № 3. - P. 106-112.

10. Fabrichnaya O., Seifert H.J. Assessment of thermodynamic functions in the $\mathrm{ZrO}_{2}-\mathrm{Nd}_{2} \mathrm{O}_{3}-\mathrm{Al}_{2} \mathrm{O}_{3}$ system // Computer Coupling of Phase Diagrams and Thermochemistry. - 2008. - V. 32. - № 1. P. 142-151. URL: https://doi.org/10.1016/j.calphad.2007.07.002 (дата обращения 15.08.2021).

11. Phase equilibria and thermodynamics in the $\mathrm{Y}_{2} \mathrm{O}_{3}-\mathrm{Al}_{2} \mathrm{O}_{3}-\mathrm{SiO}_{2}-$ system / O. Fabrichnaya, H.J. Seifert, R. Weiland, T. Ludwig, F. Aldinger, A. Navrotsky // Zeitschrift für Metallkunde. - 2001.V. 92. - P. 1083-1097.

12. Estimation of the phase diagram for the $\mathrm{ZrO}_{2}-\mathrm{Y}_{2} \mathrm{O}_{3}-\mathrm{CeO}_{2}$ system / L. Li, O. Van der Biest, P. Wang, J. Vleugels, W. Chen, S. Huang // Journal of the European Ceramic Society. - 2001. - V. 21. P. 2903-2910.

13. Synthesis of a $\mathrm{NdAlO}_{3} / \mathrm{Al}_{2} \mathrm{O}_{3}$ ceramic-ceramic composite by single-source precursor CVD / M. Veith, S. Mathur, N. Lecerf, 
K. Bartz, M. Heintz, V. Huch // Chemistry of Materials. - 2000. V. 12. - № 2. - P. 271-274. URL: https://doi.org/10.1021/ cm991149g (дата обращения 15.08.2021).

14. Shackerfold J.F., Doremus R.H. Ceramic and glass materials: structure, properties, processing. - New York: Springer, 2008. $201 \mathrm{p}$.

15. Уикс К.Е. Термодинамические свойства 65 элементов и их окислов, галогенидов, карбидов и нитридов - М.: Металлургия, 1965. $-126 \mathrm{c}$.

16. Wang Y., Liu Z.K., Chen L.Q. Thermodynamic properties of Al, $\mathrm{Ni}, \mathrm{NiAl}$ and $\mathrm{Ni}_{3} \mathrm{Al}$ from first-principles calculations // Acta Material. - 2004. - V. 52. - P. 2665-2671.

17. Greenwood N., Parish R., Thornton P. Some reaction of metal borides // Journal of the Chemical Society. - 1985. - V. 66. P. 545-549.

18. Кугай Л.Н., Назарчук Е.Н. Химическая устойчивость боридов переходных металлов // Порошковая металлургия. - 1971. № 3. - С. 51-56.

19. Модылевская К.Д., Самсонов Г.В. Стойкость боридов переходных металлов против действия кислот и щелочей // Украинский Химический Журнал. - 1985. - Т. 25. - С. 55-59.
20. Осинцев О.Е. Металловедение тугоплавких металлов и сплавов на их основе. - М.: Машиностроение, 2021. - 156 с.

21. Mojaki S.C., Mishra S.B., Mishra A.K. Synthesis, characterization and material properties of titanium carbide nanocomposite derived from biochar // Materials Letters. - 2020 - V. 264. - 5 p. URL: https://doi.org/10.1016/j.matlet.2020.127317 (дата обращения 15.08.2021).

22. Solid-state synthesis of zirconium nitride and hafnium nitride powders / Z. Wu, Z. Chen, L. Wang, L. Fang, T. Zhou, T. Mei, C. Zhang, Q. Li // Journal of the Ceramic Society of Japan. - 2021. V. 129. - P. 200-203. URL: https://doi.org/10.2109/jcersj2.20177 (дата обращения 15.08.2021).

23. Рогачев А.С., Мукасьян А.С. Горение для синтеза материалов. Введение в структурную макрокинетику. - М.: ФИЗМАТЛИТ, 2013. $-400 \mathrm{c}$.

24. Перспективные материалы и технологии самораспространяющегося высокотемпературного синтеза / Е.А. Левашов, А.С. Рогачев, В.В. Курбаткина, Ю.М. Максимов, В.И. Юхвид. М.: НИТУ МИСиС, 2011. - 377 c.

Поступила 13.10.2021 г.

\section{Информация об авторах}

Долматов O.Ю., кандидат физико-математических наук, доцент отделения ядерно-топливного цикла Инженерной школы ядерных технологий Национального исследовательского Томского политехнического университета.

Кузнецов M.C., кандидат технических наук, доцент отделения ядерно-топливного цикла Инженерной школы ядерных технологий Национального исследовательского Томского политехнического университета.

Семенов A.O., старший преподаватель отделения ядерно-топливного цикла Инженерной школы ядерных технологий Национального исследовательского Томского политехнического университета. 
UDC 621.039 .7

\title{
DEVELOPMENT OF MATRIX MATERIAL BASED ON ALUMINATE PEROVSKITE FOR IMMOBILIZATION OF ACTINIDES BY SELF-PROPAGATING HIGH-TEMPERATURE SYNTHESIS
}

\author{
Oleg Yu. Dolmatov', \\ dolmatov@tpu.ru
}

\author{
Mikhail S. Kuznetsov' ${ }^{1}$ \\ kms@tpu.ru
}

\author{
Andrey 0. Semenov', \\ semenov_ao@tpu.ru \\ 1 National Research Tomsk Polytechnic University, \\ 30, Lenin avenue, Tomsk, 634050, Russia.
}

\begin{abstract}
The relevance of the research is determined by the need to develop the method for obtaining mineral-like matrix materials intended for immobilization of the most hazardous radioactive waste of the nuclear industry. The use of the self-propagating high-temperature synthesis technology to obtain such materials is more resource-saving and does not require complex technological equipment and operations compared to traditional ways of production.

The main aim of the research is to define and propose a method for producing neodymium aluminate as a matrix material for the actinide fraction of radioactive waste.

Objects of the research are neodymium aluminate $\mathrm{NdAlO}_{3}$ with the structure of the mineral perovskite, which is used as an imitator of the trivalent fraction of radioactive waste.

Methods: evaluation and analysis of the matrix material creation by computer modeling of the self-propagating high-temperature synthesis; laboratory experiments on the synthesis of neodymium aluminate; determination of the morphological composition of the material by XDR phase analysis

Results. The paper describes a theoretical and simulation model for determining the fundamental possibility of the self-propagating hightemperature synthesis of a matrix material based on neodymium aluminate, intended for immobilization of high-level radioactive waste. Numerical simulation methods allowed us to consider various synthesis reactions of such material and determine the maximum possible amount of inclusion of a trivalent actinide simulator. Thus, it is necessary to use the reaction of SH-synthesis of aluminum-nickel with an additional content of no more than 46 wt. \% of $\mathrm{Nd}_{2} \mathrm{O}_{3}-\mathrm{Al}_{2} \mathrm{O}_{3}$ (oxide component ratio of 3,3:1) system in the initial mixture to obtain $\mathrm{NdAlO}_{3}$. The experimental study was carried out on the synthesis of the matrix material based on the results of the developed model. The authors have determined the optimal conditions for preparation of the components batch: the initial system density should not exceed 5,29 $\mathrm{g} / \mathrm{cm}^{3}$ which corresponds to pressure of $40 \mathrm{MPa}$; the maximum possible inclusion of $\mathrm{Nd}_{2} \mathrm{O}_{3}-\mathrm{Al}_{2} \mathrm{O}_{3}$ into Ni-Al system is not more than 40 wt. \%. The performed XRD analysis showed the presence of a neodymium aluminate phase in all synthesized samples; the maximum proportion of $\mathrm{NdAlO}_{3}$ is achieved during the synthesis of a sample with $40 \mathrm{wt}$. \% of $\mathrm{Nd}_{2} \mathrm{O}_{3}-\mathrm{Al}_{2} \mathrm{O}_{3}$ additive and pressure of $30 \mathrm{MPa}$. Thus, the use of a resource-efficient and simple technology of self-propagating high-temperature synthesis makes it possible to obtain a reliable matrix material for radioactive waste immobilization.
\end{abstract}

\section{Key words:}

Self-propagating high-temperature synthesis, perovskite, nuclear waste, immobilization, actinides.

\section{REFERENCES}

1. Zhang J. Nuclear fuel reprocessing and waste management. Singapore, World Scientific Publishing Company, 2018. 279 p. Available at: https://doi.org/10.1142/11017 (accessed 15 August 2021).

2. Theodore M.K., Theodore L. Introduction to environmental management. Boca Raton, CRC Press, 2021. 556 p. Available at: https://doi.org/10.1201/9781003171126 (accessed 15 August 2021).

3. Colburn H.A., Peterson R.A. A history of Hanford tank waste, implications for waste treatment, and disposal. Environmental Progress and Sustainable Energy, 2021, vol. 40, 12 p. Available at: https://doi.org/10.1002/ep.13567 (accessed 15 August 2021).

4. Solberg-Johansen B. Life cycle assessment of the nuclear fuel cycle. London, Academic Press, 1998. 349 p.

5. Ma J., Fang Z., Yang X., Wang B., Luo F., Zhao X., Wang X., Yang Y. Investigating hollandite-perovskite composite ceramics as a potential waste form for immobilization of radioactive cesium and strontium. Journal of Materials Science, 2021, vol. 56, pp. 9644-9654. Available at: https://doi.org/10.1007/s10853-02105886-2 (accessed 15 August 2021).

6. Zhoua L., Lia F., Liu J., Sun S., Liang Y., Zhang G. High-entropy $\mathrm{A}_{2} \mathrm{~B}_{2} \mathrm{O}_{7}$-type oxide ceramics: a potential immobilising matrix for high-level radioactive waste. Journal of Hazardous Materials, 2021, vol. 415,9 p. Available at: https://doi.org/10.1016/ j.jhazmat.2021.125596 (accessed 15 August 2021).

7. Barinova T.V., Podbolotov K.B., Borovinskaya I.P., Shchukin A.S Self-propagating high-temperature synthesis of ceramic matrices for immobilization of actinide-containing wastes. Radiochemistry, 2014, vol. 56, pp. 554-559. Available at: https://doi.org/10.1134/ S1066362214050178 (accessed 15 August 2021).

8. Gaidar S.M., Zhigarev V.D., Volkov A.A., Karelina M.Yu. Production of powder refractory products of self-propagating hightemperature synthesis in continuous combustion mode. Russian Metallurgy, 2017, no. 7, pp. 37-41. In Rus.

9. Mansurov Z.A., Mofa N.N., Sadykov B.S., Shabanova T.A. Activation of the technological combustion process of oxide systems by different modifying additives. Ceramic Engineering and Science Proceedings, 2013, vol. 2, no. 3, pp. 106-112.

10. Fabrichnaya O., Seifert H.J. Assessment of thermodynamic functions in the $\mathrm{ZrO}_{2}-\mathrm{Nd}_{2} \mathrm{O}_{3}-\mathrm{Al}_{2} \mathrm{O}_{3}$ system. Computer Coupling of Phase Diagrams and Thermochemistry, 2008, vol. 32, no. 1, pp. 142-151. Available at: https://doi.org/10.1016/j.calphad. 2007.07.002 (accessed 15 August 2021)

11. Fabrichnaya O., Seifert H.J., Weiland R., Ludwig T., Aldinger F., Navrotsky A. Phase equilibria and thermodynamics in the $\mathrm{Y}_{2} \mathrm{O}_{3}-$ 
$\mathrm{Al}_{2} \mathrm{O}_{3}-\mathrm{SiO}_{2}$-system. Zeitschrift für Metallkunde, 2001, vol. 92, pp. $1083-1097$.

12. Li L., Van der Biest O., Wang P., Vleugels J., Chen W., Huang S. Estimation of the phase diagram for the $\mathrm{ZrO}_{2}-\mathrm{Y}_{2} \mathrm{O}_{3}-\mathrm{CeO}_{2}$ system. Journal of the European Ceramic Society, 2001, vol. 21, pp. 2903-2910.

13. Veith M., Mathur S., Lecerf N., Bartz K., Heintz M., Huch V. Synthesis of a $\mathrm{NdAlO}_{3} / \mathrm{Al}_{2} \mathrm{O}_{3}$ ceramic-ceramic composite by single-source precursor CVD. Chemistry of Materials, 2000 vol. 12, no. 2, pp. 271-274. Available at: https://doi.org/10.1021/ cm991149g (accessed 15 August 2021).

14. Shackerfold J.F., Doremus R.H. Ceramic and glass materials: structure, properties, processing. New York, Springer, 2008. $201 \mathrm{p}$.

15. Uiks K.E. Termodinamicheskie svoistva 65 elementov i ikh okislov, galogenidov, karbidov $i$ nitridov [Thermodynamic properties of 65 elements and their oxides, halides, carbides and nitrides]. Moscow, Metalurgia Publ., 1965. 126 p.

16. Wang Y., Liu Z.K., Chen L.Q. Thermodynamic properties of Al, $\mathrm{Ni}, \mathrm{NiAl}$ and $\mathrm{Ni}_{3} \mathrm{Al}$ from first-principles calculations. Acta Material, 2004, vol. 52, pp. 2665-2671.

17. Greenwood N., Parish R., Thornton P. Some reaction of metal borides. Journal of the Chemical Society, 1985, vol. 66, pp. 545-549.

18. Kugai L.N., Nazarchuk E.N. Chemical stability of diborides of the transition metals from groups IV-V of the periodic system. Soviet powder metallurgy and metal ceramics, 1971, no. 3, pp. 212-215. In Rus.
19. Modylevskaya K.D., Samsonov G.V. Stoykost boridov perekhodnykh metallov protiv deystviya kislot i shchelochey [Resistance of transition metal borides against acids and alkalis]. Ukrainskiy Khimicheskiy Zurnal, 1985, vol. 25, pp. 55-59.

20. Osintsev O.E. Metallovedenie tugoplavkikh metallov $i$ splavov na ikh osnove [Metallurgy of refractory metals and alloys based on them]. Moscow, Mashinostroenie Publ., 2021. 156 p.

21. Mojaki S.C., Mishra S.B., Mishra A.K. Synthesis, characterization and material properties of titanium carbide nanocomposite derived from biochar. Materials Letters, 2020, vol. 264, 5 p. Available at: https://doi.org/10.1016/j.matlet.2020.127317 (accessed 15 August 2021).

22. Wu Z., Chen Z., Wang L., Fang L., Zhou T., Mei T., Zhang C., Li Q. Solid-state synthesis of zirconium nitride and hafnium nitride powders. Journal of the Ceramic Society of Japan, 2021, vol. 129, pp. 200-203. Available at: https://doi.org/10.2109/jcersj2.20177 (accessed 15 August 2021)

23. Rogachev A.S., Mukasyan A.S. Gorenie dlya sinteza materialov. Vvedenie $v$ strukturnuyu makrokinetiku [Combustion for the synthesis of materials. Introduction to structural macrokinetics]. Moscow, FIZMATLIT Publ., 2013. 400 p.

24. Levashov E.A., Rogachev A.S., Kurbatkina V.V., Maksimov Yu.M., Yuhvid V.I. Perspektivnye materialy $i$ tekhnologii samorasprostranyayushchegosya vysokotemperaturnogo sinteza [Advanced materials and technologies for self-propagating hightemperature synthesis]. Moscow, NITU MISiS, 2011.377 p.

Received: 13 October 2021.

\section{Information about the authors}

Oleg Yu. Dolmatov, Cand. Sc., associate professor, National Research Tomsk Polytechnic University.

Mikhail S. Kuznetsov, Cand. Sc., associate professor, National Research Tomsk Polytechnic University.

Andrey O. Semenov, assistant, National Research Tomsk Polytechnic University. 